\title{
Osteoporosis: An Age-Related and Gender-Specific Disease - A Mini-Review
}

\author{
Peter Pietschmann ${ }^{a, b}$ Martina Rauner ${ }^{b, c}$ Wolfgang Sipos ${ }^{d}$ \\ Katharina Kerschan-Schindle \\ a Department of Pathophysiology, Center for Physiology, Pathophysiology and Immunology, Medical University of \\ Vienna, and ${ }^{b}$ Ludwig Boltzmann-Institute of Aging Research, Vienna, Austria; ${ }^{C}$ Division of Endocrinology, \\ Diabetes and Bone Diseases, Department of Medicine III, Technical University, Dresden, Germany; \\ ${ }^{d}$ 2nd Medical Clinic, University of Veterinary Medicine Vienna, and ${ }^{\mathrm{e}}$ Department of Physical Medicine \\ and Rehabilitation, Medical University of Vienna, Vienna, Austria
}

\section{Key Words}

Osteoporosis • Fractures $\cdot$ Bone remodeling $\cdot$ Osteoblasts .

Osteoclasts $\cdot$ Gender medicine

\begin{abstract}
Osteoporosis, a classical age-related disease and known to be more common in women than in men, has been reported increasingly often in men during the past few years. Although men at all ages after puberty have larger bones than women, resulting in greater bending strength, mortality after a hip fracture, one of the major complications of osteoporosis, is more common in men than in women. Sex hormone deficiency is associated with unrestrained osteoclast activity and bone loss. Even though estrogen deficiency is more pronounced in women, it appears to be a major factor in the pathogenesis of osteoporosis in both genders. In contrast to osteoporosis in postmenopausal women, the treatment of osteoporosis in men has been scarcely reported. Nevertheless, some drugs commonly used for the treatment of osteoporosis in women also appear to be effective in men. The aim of this study is to review primary osteoporosis in the elderly with particular emphasis on gender-related aspects.

Copyright $\odot 2008$ S. Karger AG, Basel
\end{abstract}

\section{Introduction}

Osteoporosis is one of the most common conditions associated with aging. It is defined as a skeletal disorder characterized by compromised bone strength predisposing a person to an increased risk of fracture. Bone strength primarily reflects the integration of bone density and bone quality [1]. The risk of fracture increases 1.5- to 3fold or more for each standard deviation (SD) decrease in bone mineral density (BMD). A BMD value between 1 and 2.5 SD below the mean value for young adults is defined as low bone mass or osteopenia. A person with a BMD more than 2.5 SD below the adult mean value is considered to suffer from osteoporosis. This classification has been established for lumbar spine bone mineral densities of postmenopausal women only. Nevertheless, the above-mentioned diagnostic categories are used for men as well as women.

Many risk factors contribute to the pathogenesis of osteoporosis. Age-specific variations in bone density are mainly determined by genetic factors. Nevertheless, lifestyle factors such as bone-loading physical activities, nutrition (calcium and vitamin D intake, alcohol consumption), nicotine abuse, illnesses or the intake of medica-

\section{KARGER}

Fax +41613061234 E-Mail karger@karger.ch www.karger.com
Dr. Peter Pietschmann, MD

Department of Pathophysiology, Center of Physiology and Pathophysiology

Medical University of Vienna, Währinger Gürtel 18-20, AT-1090 Vienna (Austria)

Tel. +43 140400 5122, Fax +43 1404005130

E-Mail peter.pietschmann@meduniwien.ac.at 
tions with a negative impact on bone metabolism are additional risk factors for osteoporosis.

The aim of this review is to describe the epidemiology, pathophysiology, biomechanics, and therapy of osteoporosis from the perspective of gender-related differences.

\section{Epidemiology and Clinical Relevance}

The lifetime risk for a fragility fracture (distal forearm fracture, hip fracture, clinical vertebral fracture) in a 50year-old white US woman has been estimated to be approximately $40 \%$, whereas the risk for a white US man is $13 \%$ [2]. In this study, the risk of a clinical vertebral fracture was three times higher in women than in men. However, in the European Vertebral Osteoporosis Study [3] and the Canadian Multicenter Osteoporosis Study [4], the prevalence of vertebral deformities was similar in men and women. The occurrence of fractures was found to increase with age in both sexes. Nevertheless, the curve was steeper in women than in men.

An analysis of risk factors in women and men with a clinical fracture showed a similar prevalence of bone-related risk factors in both genders, except for low body weight and a previous fracture after the age of 50, which are more common in women [5]. Men experience fractures at higher bone mineral density values than do women.

Osteoporotic fractures lead to pain and occasional disability. More importantly, they increase mortality. Mortality rates after hip fracture are 4 -fold higher in men than in women [6]. A vertebral fracture may be regarded as a risk factor for subsequent long-term morbidity, especially in women, and for mortality in both genders. The costs of treating fractures in women are higher than those for men.

\section{Pathophysiology}

\section{Bone Biology and Aging}

Bone is a very active tissue that is remodeled throughout life. The purpose of bone remodeling is to repair microdamage, adapt the skeleton to mechanical loading, and maintain calcium and phosphorus homeostasis. Bone remodeling is the consequence of a coupled action of bone-resorbing cells (osteoclasts) and bone-forming cells (osteoblasts). The bone remodeling cycle starts with the degradation of old bone by osteoclasts, which requires about 2-4 weeks. Osteoblasts then synthesize organic ex- tracellular bone matrix (osteoid) and orchestrate its mineralization. Bone-forming processes take about 4-6 months. After the formation and mineralization of new bone, the bone remodeling unit enters a resting phase.

Osteoblasts, as adipocytes, fibroblasts, chondroblasts and myoblasts, differentiate from mesenchymal stem cells. After bone formation osteoblasts convert into inactive lining cells, terminally differentiate into osteocytes, or undergo apoptosis. In contrast to osteoblasts, osteoclasts are derived from the hematopoietic lineage and can be generated from mononuclear precursors. A highly coordinated communication between bone forming and bone-degrading cells is needed to maintain bone homeostasis. Two cytokines - mainly produced by bone marrow stromal cells and osteoblasts - are essential and adequate for osteoclast generation: macrophage colony-stimulating factor (M-CSF), and receptor activator of nuclear factor-kappa B ligand (RANKL). RANK, a receptor for RANKL, is expressed on osteoclast precursors. RANKLRANK interactions stimulate the differentiation and activation of osteoclasts and inhibit osteoclast apoptosis. These processes are antagonized by osteoprotegerin, a natural decoy receptor of RANKL also mainly produced by stromal cells and osteoblasts [for review, see 7]. These interactions are already under clinical investigation with regard to their potential therapeutic application [reviewed in 8]. Whereas the RANKL/RANK/osteoprotegerin system mediating osteoblast-osteoclast coupling was identified as early as 10 years ago, signaling molecules for osteoclast-osteoblast communication were only recently discovered. Osteoclasts express ephrinB2, whose receptor ephB4 is expressed by osteoblasts [9]. The interaction between ephrinB2 and ephB4 appears to limit osteoclast activity and promote osteoblast differentiation.

Generally speaking, osteoporosis results from any imbalance between the activity of osteoblasts and that of osteoclasts, which leads to bone degradation. With regard to aspects of bone formation, a number of experimental and clinical studies have been published on the effect of age on the generation, differentiation and function of osteoblasts. Most - but not all - investigators registered a decrease in the osteoprogenitor pool of bone marrow with advancing age [10]. Decreased osteoblastogenesis is also seen in the senescence-accelerated mouse P6 (SAMP6), a model of involutional osteoporosis associated with spontaneous fractures in old age [11].

An important and characteristic feature of the aging bone marrow is a shift from osteoblastogenesis to adipogenesis, leading to the accumulation of bone marrow fat. Runx2 is the first osteoblast-specific transcription factor 
Fig. 1. Gene expression of runx2 and alkaline phosphatase (ALP) decrease in bone tissue and in osteoblasts with age. a Bone marrow was flushed out of the femora of young (6-weeks) and old (18-months) $\mathrm{BALBC}$ mice. The bone tissue was crushed in liquid nitrogen. RNA was isolated and subjected to real-time PCR analysis for runx 2 and ALP. Gene expression was normalized to GAPDH. b Bone marrow stromal cells were isolated from the bone marrow of young ( 6 weeks) and old (18 months) mice and cultured for 21 days under osteoinductive conditions. RNA was isolated and subjected to real-time PCR analysis for runx2 and ALP. Gene expression was normalized to GAPDH. ${ }^{*} \mathrm{p}<0.01$.
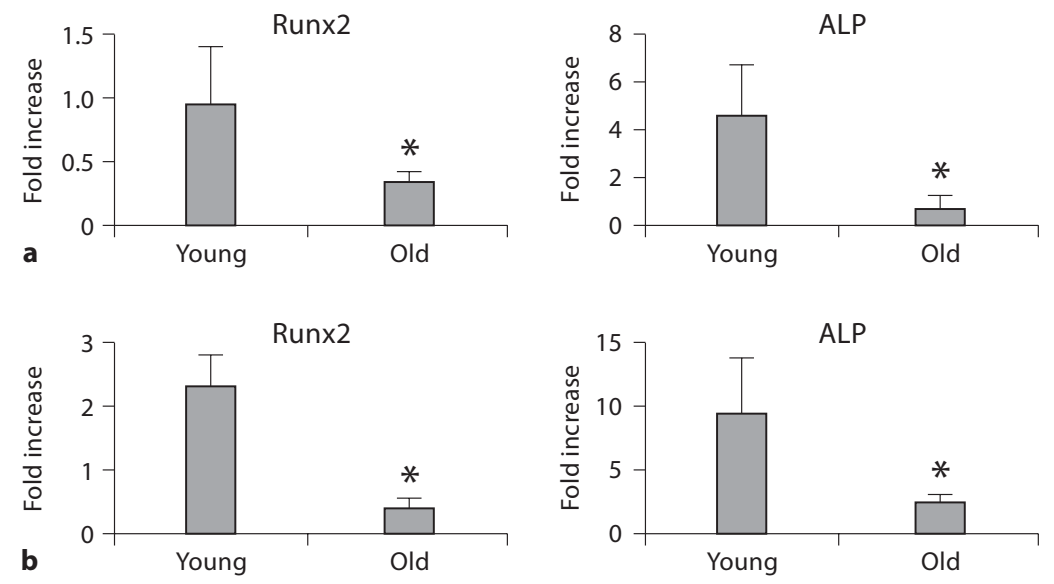

that was described. Runx2-deficient mice completely lack ossification because of the absence of osteoblast differentiation [12]. In contrast, peroxisome-proliferatoractivated receptor $\gamma 2$ (PPAR $\gamma 2$ ) inhibits osteoblast but promotes adipocyte proliferation. Several studies indicate that the expression of runx 2 in osteoblasts decreases with age (fig. 1), while that of PPAR $\gamma 2$ increases [13]. Thus, alterations in these transcription factors appear to explain - at least in part - the age-related shift from osteoblastogenesis to adipogenesis, and thus the transition from red to yellow bone marrow.

More insights into age-related process especially in bone can be appreciated from the pathogenesis of the Hutchinson-Gildford progeria syndrome. This is a disease caused by a mutation in the lamin $\mathrm{A} / \mathrm{C}$ gene and is characterized by premature aging, including atherosclerosis, alopecia and osteoporosis with spontaneous fractures [14].

Lamins are intermediate filaments that are important constituents of the inner nuclear membrane, and its expression has been found to decrease in osteoblasts of aged mice. Very recent data from our group indicate that knock-down of lamin A/C in human mesenchymal stem cells leads to a decrease of osteoblast proliferation, a reduction of runx 2 mRNA expression and an increase in the RANKL/OPG ratio [15]. Thus, these findings indicate a novel link between lamin A/C deficiency and age-related osteoblast insufficiency, a feature that is also seen in klotho-deficient mice (which exhibit many pathologies resembling human aging).

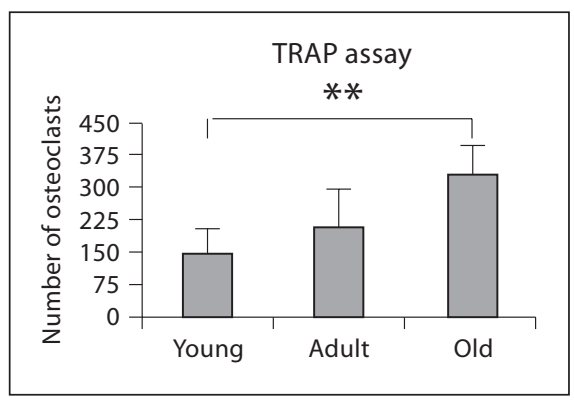

Fig. 2. Number of osteoclasts increases in old mice. Osteoclasts were derived from murine $(\mathrm{C} 57 \mathrm{BL} / 6 \mathrm{~J})$ bone marrow stromal cells and were cultured for 7 days in the presence of $10 \mathrm{nM}$ 1,25-dihydroxyvitamin $\mathrm{D}_{3}$. Cells were stained for tartrate-resistant acid phosphatase (TRAP). Multinucleated, TRAP-positive cells were counted as osteoclasts. ${ }^{* *} \mathrm{p}<0.01$.

With regard to the effects of aging on osteoclast generation, contradictory data have been reported in the published literature. Perkins et al. [16] suggested that agerelated bone loss in mice is associated with an increase in the osteoclast progenitor pool, and that bone marrow cells from aged mice exhibit a greater ability to form osteoclasts. A greater capacity for osteoclast generation in mice was also registered by Cao et al. [17], as well as our group (fig. 2), and was found to be related to increased mRNA expression of RANKL. In contrast, osteoclastogenesis is impaired in senescence-accelerated mice, possibly due to osteoblast insufficiency [11]. Data from our laboratory [18] show that, in aged male rats, serum 
Fig. 3. Number of RANKL-expressing $T$ lymphocytes and expression of RANKL on $\mathrm{T}$ lymphocytes decrease in old mice. Peripheral mononuclear cells were isolated from the spleen of young (6 weeks), adult (6 months) and aged C57BL/6J mice (18 months), stained with anti-RANKL (PE, eBioscience) and anti-CD4 (FITC, $\mathrm{BD})$ and anti-CD8 (PerCP, BD), respectively, and were subjected to flow cytometric analysis. Surface and intracellular expression of RANKL (a) and mean fluorescence intensity (b) on CD4- and CD8-positive T cells. ${ }^{* *} \mathrm{p} \leq 0.01,{ }^{* *} \mathrm{p} \leq$ 0.005 .

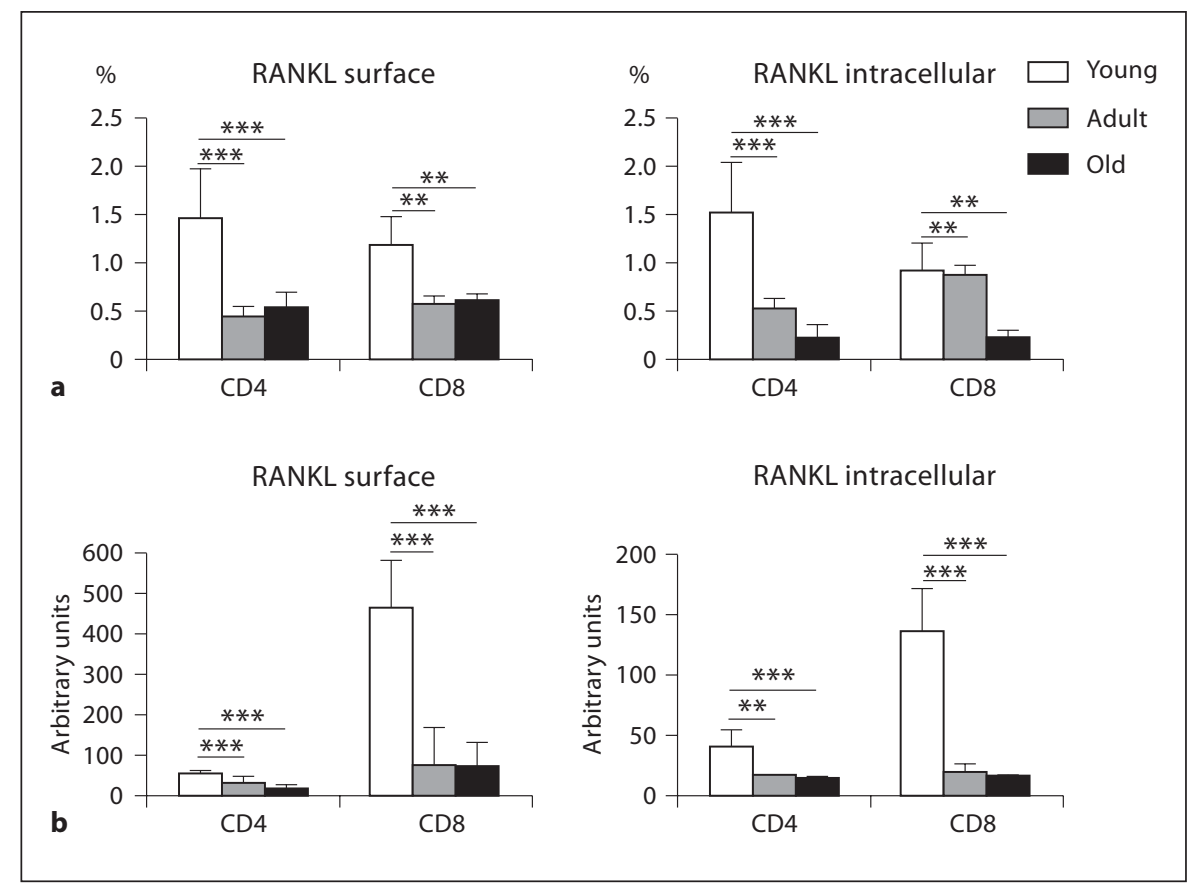

RANKL levels and ex vivo osteoclast generation are diminished despite demonstrable evidence of increased bone resorption. A decline in serum RANKL levels with age has also been registered in human studies [19] and is in line with the age-related decline of RANKL expression in T cells (fig. 3). Thus, data on effects of aging on RANKL expression, and osteoclast generation as well, are contradictory.

Alternative approaches to investigate changes in the number and/or activity of cells involved in bone remodeling are bone histomorphometry and the determination of biochemical markers of bone turnover. Most, but not all authors who used bone histomorphometry registered a decline in bone formation with age $[11,20]$. In men, serum levels of markers of bone formation (such as osteocalcin or bone-specific alkaline phosphatase) decline until the 6 th decade. Older men, on the other hand, were shown to demonstrate stable or increased as well as decreased bone formation markers $[21,22]$. A considerable number of studies have reported an increase in markers of bone formation and bone resorption in postmenopausal women compared to premenopausal women [21], reflecting a high remodeling rate on histomorphometry. In older men, bone degradation as determined by biochemical markers appears to increase [21,22]. Thus, particularly with regard to bone formation, age-related changes in bone remodeling appear to be gender specific.

\section{A Model of Involutional Osteoporosis}

In 1983, Riggs and Melton [23] proposed the existence of two distinct types of involutional osteoporosis: postmenopausal (type 1) and senile (type 2) osteoporosis. Type 1 osteoporosis refers to the rapid phase of bone loss observed approximately 5-10 years after menopause, involves mainly trabecular bone, and is manifested clinically by fractures of the distal radius and vertebrae. While postmenopausal osteoporosis was recognized to be caused by estrogen deficiency, senile osteoporosis was attributed to aging processes such as osteoblast dysfunction. Type 2 osteoporosis involves both trabecular and cortical bone. In addition to vertebral fractures, hip fractures are also characteristic for this type of osteoporosis.

In 1998 the typel/type 2 model was reformulated as a 'unitary model of osteoporosis in postmenopausal women and aging men' [23]. In the unitary model, estrogen deficiency was deemed to be the main cause of bone loss in early and also late postmenopausal women and elderly men. Estrogens have multiple effects on bone cells and mineral homeostasis. Estradiol inhibits the generation and activity of osteoclasts [24], effects mediated by an upregulation of osteoprotegerin. As mentioned above, estrogen withdrawal results in a high remodeling rate with excessive bone resorption and rapid bone loss. EghbaliFatourechi et al. [25] showed that upregulation of RANKL 
in the bone marrow is an important mediator of high bone degradation in early postmenopausal women.

In addition to its effect on the RANKL/osteoprotegerin system, estrogen deficiency leads to dysregulation of $\mathrm{T}$ cell function and antigen presentation. Pacifici et al. [26] demonstrated that estrogen withdrawal results in increased production of interleukin-7 (in part due to a decrease in transforming growth factor- $\beta$ ), leading to $\mathrm{T}$ cell activation and greater release of interferon- $\gamma$ by $\mathrm{T}$ cells. Interferon- $\gamma$ upregulates the expression of major histocompatibility complex class II molecules, which results in augmented antigen presentation by bone marrow macrophages and dendritic cells. Consequently, T cells are further activated and enhance the production of RANKL and tumor necrosis factor- $\alpha$, which results in increased osteoclastogenesis [26]. Notably, a progressive proinflammatory status has not only been associated with the postmenopausal phase but also with aging in general; this phenomenon has been referred to as 'inflamm-aging' [27]. Data from our laboratory suggest that some phenomena of 'inflamm-aging' are gender specific: interferon $-\gamma$ production by $\mathrm{T}$ cells increased with age in women, but not in men [22].

The rapid phase of postmenopausal bone loss approximately 5-10 years after menopause is followed by a slower phase of continuous bone loss ('senile bone loss'). Notably, extraskeletal consequences of estrogen deficiency (such as reduced intestinal calcium absorption) lead to a progressive elevation of PTH and further stimulate bone degradation [23].

Although testosterone deficiency in men is clearly associated with bone loss, estrogens appear to be even more important than androgens for male bones. This notion derives from several lines of evidence: the description of a mutated estrogen receptor gene in a young man with osteoporosis, observational studies demonstrating that estrogen levels correlate better than testosterone levels with bone mineral density [28], and intervention studies in elderly men showing a greater suppressive effect of estradiol on bone resorption than testosterone [28]. In men with idiopathic osteoporosis, estradiol levels are decreased, whereas sex-hormone-binding globulin (SHBG) levels and bone resorption markers are elevated [29]. SHBG levels in men increase with aging. High SHBG levels could significantly contribute to the development of osteoporosis by decreasing the bioavailability of estradiol (and testosterone), thus facilitating unrestrained osteoclast activity that exceeds bone formation. Recently, it has been suggested that the increase of serum SHBG is due to complex interactions between the reduction of pituitary growth hormone secretory capacity and decreased testosterone production by the testes [28]. Despite the fact that estrogens are crucially important for bone health in women and men, it should be borne in mind that - in contrast to the abrupt cessation of ovarian function during menopausal transition - there is a slight but steady decrease in estradiol and testosterone levels with aging in men.

Apart from estrogen deficiency, vitamin D deficiency is a major factor accounting for bone fragility in the elderly. It is well established that vitamin D levels decrease with aging [22]; in particular in residents of nursing homes, extremely low concentrations of vitamin D have been reported. If vitamin D levels are very low, the clinical picture of osteomalacia may develop; the concomitant existence of osteoporosis and osteomalacia has been referred to as 'osteoporomalacia'. Low levels of vitamin D in elderly subjects may result from a lack of sunlight exposure, inadequate dietary intake, or medical conditions such as renal insufficiency. One of the major effects of the active metabolite of vitamin D, i.e. $1,25(\mathrm{OH})_{2}$ vitamin $\mathrm{D}$, is to increase the intestinal absorption of calcium and phosphate. Thus, in vitamin D deficiency intestinal calcium absorption is decreased and may lead to hypocalcemia; as a consequence PTH levels increase. High PTH levels result in enhanced bone degradation which on the one hand counteracts hypocalcemia, but on the other hand in the long run leads to bone loss.

As will be discussed in more detail later, frequent falls in elderly subjects clearly increase the risk of fractures. Vitamin D receptors are not only expressed in bone but also in other tissues, including skeletal muscle. Vitamin $\mathrm{D}$ deficiency has been associated with muscle weakness and an increased frequency of falls; accordingly, vitamin D supplementation may reduce the risk of falling [1].

\section{Biomechanics}

Low bone mass is not the only factor contributing to the frequency of fractures in the aging population. As early as 20 years ago it was shown that, for a given bone mass, the risk of fractures increases with age, indicating the existence of other factors besides bone mass that predispose an individual to sustain a nontraumatic fracture.

Factors most likely to influence the resistance to fracture include the overall composition (i.e. proportion of mineral, collagen, water and matrix proteins), the physical and biochemical characteristics of these components 


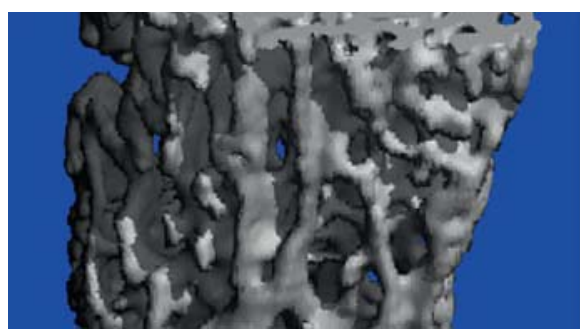

Young (6 weeks)

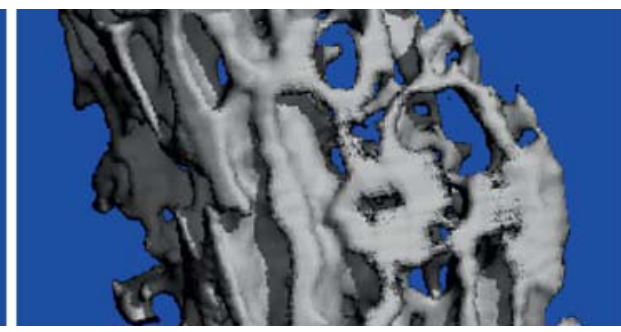

Adult (6 months)

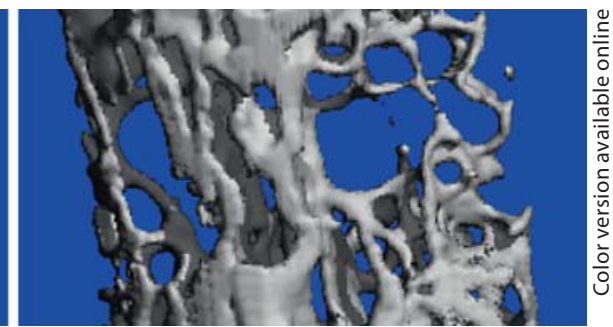

Old (18 months)

Fig. 4. Bone mass is decreased in the vertebral column of aged mice. Representative $\mu \mathrm{CT}$ images of the fourth vertebral body of young ( 6 weeks), adult (6 months) and old (18 months) C57BL/6J mice.

(i.e. nature of collagen, degree of mineralization), the morphology and architecture (i.e. bone size, geometry, trabecular microarchitecture), and the severity and nature of pre-existing microdamage (i.e. crack length and location) [30]. One example of trabecular bone loss and trabecular architectural disruption with aging is shown in figure 4. A balance between the mineral stiffness of bone and its flexibility is achieved by varying its mineral content [31]. The greater the mineral content, the greater the material stiffness and the lesser its flexibility. The association between areal BMD and vertebral fracture risk is explained by its correlation with more specific parameters of bone density, structure, and strength. Besides, bone structure, strength, and load-strength ratios contribute to forearm fracture risk independently of areal BMD [32].

As long as bone grows in length, periosteal apposition increases in diameter while concurrent endocortical resorption excavates the marrow cavity. There is little difference between the genders with regard to the extent of periosteal apposition and net endocortical remodeling until puberty [33]. Estrogen in girls inhibits periosteal bone formation, limiting the diameter of bone and simultaneously promoting net bone formation on the endocortical surface. In boys, pubertal androgen production increases periosteal apposition, bone diameter, and cortical thickness. Continued widening of the inner diameter shifts the cortex even farther from the neutral axis than the displacement that occurs in girls [31]. Thus, young men have greater bending strength than do young women because bending strength increases with the distance from the neutral axis. However, cortical thickness is similar in both sexes [31]. The fact that men have larger bones at all ages compared to women [31] may explain why stresses are higher in women compared to men when simulating a fall on the greater trochanter [34]. A simu- lated investigation of forearm behavior when falling showed that women had significantly less failure strength, bone mineral content, trabecular density and cortical area than did men [35]. In premenopausal women, periosteal apposition increases the width of the radius, partly offsetting endocortical resorption [36]. During menopause, increased periosteal apposition partially preserves bone strength [36]. The postmenopausal period is characterized by a further acceleration of endocortical resorption, whereas periosteal apposition increasingly declines [36]. Thus, the estimated cortical area and bending strength decrease in the postmenopausal period.

Gender-related differences are found not only in peripheral bones. Men have greater vertebral strength at all ages, largely because of the greater cross-sectional area of vertebrae. The latter is estimated to be $25 \%$ less in women than in men [37]. According to another study, periosteal bone formation increases vertebral body cross-sectional area to a 3 -fold greater extent in men than in women, distributing loads over a larger cross-sectional area (CSA) so that the load imposed per unit CSA decreased twice as much in men than in women [37]. The fact that women generally experience greater relative loads is in accordance with other studies [38]. The age-associated decline in vertebral strength is greater in women than men. In women, the ultimate load of the spine and the fracture risk score (i.e. quotient of estimated spinal load and estimated ultimate strength) begin to decrease significantly after the fifth decade, whereas this occurs in men after the sixth decade [39]. For bending and lifting, the risk of vertebral fractures, defined as the ratio of spinal load to vertebral strength, exceeded 1.0 in $30 \%$ of women and $12 \%$ of men aged at least 50 years [40].

Loading factors include the incidence and mechanics of falls, which occur more frequently in the elderly. One third of community-dwelling individuals older than 65 
years of age experience falls every year. Obviously, the more times an individual falls, the greater is the risk of fracture. Hip fractures are almost always due to a fall, but only $5 \%$ of falls result in fractures [41]. The risk of hip fractures is greatest for a sideways fall that impacts on the greater trochanter. In such a case, the risk of hip fracture has been shown to rise up to 6-fold [42]. Disturbance type and gait speed significantly affect the direction of a fall and location of the impact. Slipping or fainting while walking slowly is more likely to result in an impact on the hip, suggesting a greater risk of hip fracture [43]. In the latter study, four types of disorders (faint, slip, step down and trip) were investigated at fast, normal and slow speed. A meta-analysis showed that a low body mass index is a risk factor for hip fracture even after adjustment for femoral areal BMD [44]. In women, a threefold increase in soft tissue thickness has been shown to reduce predicted peak force by $35 \%$ [45]. In a recent investigation of human cadaveric specimens, it was found that each SD decrease in trochanteric soft tissue thickness was associated with a 1.8-fold increase in the risk of hip fracture [46].

\section{Treatment}

\section{Osteoporosis-Specific Medication}

Although there is still some discussion on the optimal dose, calcium and vitamin D supplementation is widely accepted as a fundamental basic measure for the prevention and treatment of osteoporosis. However, large clinical trials conducted over the past 15 to 20 years clearly established that - in addition to calcium and vitamin D - patients with osteoporosis should receive specific pharmacologic treatment. In 2005, Riggs and Parfitt [47] proposed a classification of drugs used to treat osteoporosis, based on their action on bone remodeling. Anticatabolic drugs (such as bisphosphonates, or raloxifene - a selective estrogen receptor modulator) decrease bone remodeling and reduce fractures by preserving skeletal microarchitecture and moderately increasing bone mass. Anabolic drugs (such as teriparatide or PTH(1-84)), on the other hand, reduce fractures by enhancing remodeling. In addition to substantially increasing bone mass, anabolic drugs appear to repair bone microarchitecture and improve bone geometry. Strontium ranelate, which is available for the treatment of osteoporosis in Europe, does not fit into the aforementioned classification because it inhibits bone resorption and stimulates bone formation.

There is a large body of scientific evidence derived from randomized controlled trials concerning the treat- ment of osteoporosis in postmenopausal women; for a detailed discussion the reader is referred elsewhere $[1$, 48]. To summarize, a number of substances were shown to be effective against both vertebral and nonvertebral fractures: three different aminobisphosphonates (alendronate, risedronate, zoledronic acid), teriparatide, strontium ranelate and hormone replacement therapy (the lattermost is no longer recommended for the prevention or treatment of osteoporosis). Evidence of efficacy against vertebral fractures is available for ibandronate (an aminobisphosphonate), raloxifene and $\mathrm{PTH}(1-84)$.

In contrast to postmenopausal women, osteoporosis in men is scarcely investigated. In 2000, the first large controlled trial on osteoporosis treatment in men showed that alendronate increased bone mineral density and prevented vertebral fractures [49]. In men receiving glucocorticoids, risedronate reduced the risk of vertebral fractures [50]. In a randomized open label study performed in men with primary or secondary osteoporosis, Ringe et al. [51] found a significantly lower incidence of new vertebral fractures in men treated with risedronate when compared to alphacalcidol. Although the above mentioned studies constitute a rationale for the treatment of osteoporosis in men, undertreatment of elderly men with osteoporosis is very common in clinical practice. Thus, further research needs to be conducted on gender-related aspects of the treatment of osteoporosis.

\section{Training}

The public health burden of fractures cannot be resolved by drugs alone because drug trials have been largely focused on women at the highest risk of fractures due to osteoporosis. However, a large number of individuals who suffer fractures have a bone mineral density even less than $-2.5 \mathrm{SD}$ below the normal value in young adults. Therefore, one goal is to achieve an acceptable bone mineral density in the general population. Prophylaxis should be started in childhood, with the aim of increasing peak bone mass. A 7-month exercise program of gradually increasing intensity in pre- and early-pubertal girls showed greater changes in the cross-sectional area of femoral neck bone, cortical thickness, and section modulus in the intervention group than in controls [52]. According to experimental studies, the response to exercise is gender specific; the greatest effects are seen in male animals [53].

\section{Vertebroplasty/Kyphoplasty}

These methods of percutaneous cement injection provide minimally invasive options for the management of osteoporotic vertebral compression fractures. According 
to a recent review, a mean restoration of $6.6^{\circ}$ in the kyphotic angle is achieved by both procedures [54]. A large number of subjects experience some pain relief: $87 \%$ with vertebroplasty and $92 \%$ with kyphoplasty. With regard to complication rates, cement leaks occur in $41 \%$ and $9 \%$ of vertebrae treated by vertebroplasty and kyphoplasty, respectively. Following either procedure, new fractures of adjacent vertebrae occur at rates higher than those registered in the general osteoporotic population, but approximately equivalent to the general osteoporotic population that had experienced a previous vertebral fracture.

\section{Hip Protectors}

As mentioned above, the risk of hip fracture is greatest for a sideways fall that impacts on the greater trochanter [42]. An increase in the thickness of soft tissue has been shown to reduce the predicted peak force on the trochanter [45]. Thus, trochanteric padding systems designed to absorb part of the impact on the hip when falling may reduce the risk of hip fractures. However, the results of studies investigating hip protectors for prevention of hip fractures in the elderly are contradictory. Pooling data from eleven trials conducted in nursing or residential care settings, including cluster-randomized studies, demonstrated a significant reduction in the frequency of hip fractures [55]. However, pooling data from three individually randomized trials involving more than 5,000 community-dwelling persons showed no reduction in the frequency of hip fractures [55]. In line with the doubts expressed in this review concerning the effectiveness of hip protectors in the elderly, a more recent meta-analysis [56] reported limited evidence of hip protectors preventing hip fractures in care homes. Many hip fractures occur in persons with a low risk for hip fractures or under circumstances that preclude the use of hip protectors. This may be the reason why the actual preventive effect of hip protectors is lower than its maximum potential preventive effect [57]. Cost-effectiveness analyses are inconsistent in that they report positive as well as negative results. The primary acceptance of hip protectors as well as compliance rates are low.

Compliance can be raised by providing information on the dangers of hip fractures as well as improving the design and mechanical properties of hip protectors [58]. Soft-shell protectors are possibly more acceptable to users than the traditional hard-shell designs. However, in simulated sideways falls, soft-shell protectors were shown to achieve no more than a marginal reduction of the total impact force [59]. Further clinical trials are needed to clarify this issue.

\section{Conclusion}

Osteoporosis is an age-related disease with several gender-specific differences. Its prevalence is higher in women than in men. Due to their larger bones, men have greater bending strength than do women. However, once a hip fracture has occurred, mortality is higher in men. Differences in sex hormone production - especially the abrupt decline of estrogen in women - are responsible for inter-gender differences in the pathophysiology of osteoporosis. Finally, the treatment of osteoporosis also differs between genders; some therapy options can only be used in women.

\section{Acknowledgments}

This work was supported in part by the Austrian Science Fund (FWF, Grant Number P20239-B13). The authors are grateful to Prof. Dr. Philippe Zysset and Dipl. Ing. Peter Varga (Technical University of Vienna) for the microCT analyses and to Ms. Birgit Schwarz for her help with the preparation of the manuscript.

\section{Conflict of Interest Statement}

Dr. Peter Pietschmann has received research support and/or honoraria from Amgen GmbH, Eli Lilly GmbH, Merck Sharp \& Dohme Gmbh, Nycomed Pharma, Roche Austria, Servier Austria, Sanofi Aventis.

References

Pietschmann/Rauner/Sipos/

Kerschan-Schindl 
6 Cree M, Soskolne CL, Belseck E, Hornig J, Mc Elhaney JE, Brant R, Suarez-Almazor M: Mortality and institutionalization following hip fracture. J Am Geriatr Soc 2000;48:283288.

7 Rauner M, Sipos W, Pietschmann P: Osteoimmunology. Int Arch Allergy Immunol 2007; 143:31-48.

$>8$ Sipos W, Pietschmann P, Rauner M: Strategies for novel therapeutic approaches targeting cytokines and signalling pathways of osteoclasto- and osteoblastogenesis in the fight against immune-mediated bone and joint diseases. Curr Med Chem 2008;15:127-136.

9 Mundy GR, Elefteriou F: Boning up on ephrin signaling. Cell 2006;126:441-443.

10 Bergman RJ, Gazit D, Kahn AJ, Gruber H, McDougall S, Hahn TJ: Age-related changes in osteogenic stem cells in mice. J Bone Miner Res 1996;11:568-577.

11 Jilka RL, Weinstein RS, Takahashi K, Parfitt AM, Manolagas SC: Linkage of decreased bone mass with impaired osteoblastogenesis in a murine model of accelerated senescence. J Clin Invest 1996;97:1732-1740.

-12 Ducy P, Zhang R, Geoffroy V, Ridall AL, Karsenty G: Osf2/Cbfa1: a transcriptional activator of osteoblast differentiation. Cell 1997;89:747-754.

-13 Moerman EJ, Teng K, Lipschitz DA, LeckaCzernik B: Aging activates adipogenic and suppresses osteogenic programs in mesenchymal marrow stroma/stem cells: the role of PPAR-gamma2 transcription factor and TGF-beta/BMP signaling pathways. Aging Cell 2004;3:379-389.

- 14 De Sandre-Giovannoli A, Bernard R, Cau P, Navarro C, Amiel J, Boccaccio I, Lyonnet S, Stewart CL, Munnich A, Le Merrer M, Lévy $\mathrm{N}$ : Lamin a truncation in Hutchinson-Gilford progeria. Science 2003;300:2055.

15 Rauner M, Sipos W, Foisner R, Hofbauer L, Pietschmann P: Silencing lamin A/C decreases osteoblast differentiation and stimulates osteoclastogenesis through enhanced RANKL signaling. Calcified Tissue Int 2008; 82:50-51.

16 Perkins SL, Gibbons R, Kling S, Kahn AJ: Age-related bone loss in mice is associated with an increased osteoclast progenitor pool. Bone 1994;15:65-72.

$\checkmark 17$ Cao J, Wronski TJ, Iwaniec U, Phleger L, Kurimoto P, Boudignon B, Halloran BP: Aging increases stromal/osteoblastic cell-induced osteoclastogenesis and alters the osteoclast precursor pool in the mouse. J Bone Miner Res 2005;20:1659-1668.

-18 Pietschmann P, Skalicky M, Kneissel M, Rauner M, Hofbauer G, Stupphann D, Viidik A: Bone structure and metabolism in a rodent model of male senile osteoporosis. Exp Gerontol 2007;42:1099-1108.
19 Kerschan-Schindl K, Wendlova J, Kudlacek S, Gleiss A, Woloszczuk W, Pietschmann P: Serum levels of receptor activator of nuclear factor kappaB ligand (RANKL) in healthy women and men. Exp Clin Endocrinol Diabetes 2008;116:491-495.

20 Recker RR, Kimmel DB, Parfitt AM, Davies KM, Keshawarz N, Hinders S: Static and tetracycline-based bone histomorphometric data from 34 normal postmenopausal females. J Bone Miner Res 1988;3:133-144.

21 Szulc P, Delmas PD: Biochemical markers of bone turnover in osteoporosis; in Marcus R, Feldman D, Nelson DA, Rosen CF (eds): Osteoporosis, ed 3. Amsterdam, Elsevier, 2008, vol 2, pp 1519-1545.

22 Pietschmann P, Gollob E, Brosch S, Hahn P, Kudlacek S, Willheim M, Woloszczuk W, Peterlik M, Tragl KH: The effect of age and gender on cytokine production by human peripheral blood mononuclear cells and markers of bone metabolism. Exp Gerontol 2003;38:1119-1127.

23 Riggs BL, Khosla S, Melton LJ 3rd: A unitary model for involutional osteoporosis: estrogen deficiency causes both type I and type II osteoporosis in postmenopausal women and contributes to bone loss in aging men. J Bone Miner Res 1998;13:763-773.

24 Schiller C, Gruber R, Redlich K, Oberhollenzer F, Weger S, Egger G, Mayr A, Jocher J, Xu Q, Pietschmann P, Teitelbaum S, Smolen J, Willeit J: 17 $\beta$-Estradiol antagonizes effects of $1 \alpha, 25$-dihydroxyvitamin $\mathrm{D}_{3}$ on interleukin- 6 production and osteoclast-like cell formation in mouse bone marrow primary cultures. Endocrinology 1997;138:4567-4571.

25 Eghbali-Fatourechi G, Khosla S, Sanyal A, Boyle WJ, Lacey DL, Riggs BL: Role of RANK ligand in mediating increased bone resorption in early postmenopausal women. J Clin Invest 2003;111:1120-1122.

26 Pacifici R: Postmenopausal osteoporosis: how the hormonal changes of menopause cause bone loss; in Marcus R, Feldman D, Nelson DA, Rosen CF (eds): Osteoporosis, ed 3 . Amsterdam, Elsevier, 2008, vol 2, pp 10411054.

27 Franceschi C, Bonafè M, Valensin S, Olivieri F, De Luca M, Ottaviani E, De Benedictis G: Inflamm-aging: an evolutionary perspective on immunosenescence. Ann NY Acad Sci 2000;908:244-254.

28 Riggs BL, Khosla S, Melton J 3rd: Estrogen, bone homeostasis, and osteoporosis; in Marcus R, Feldman D, Nelson DA, Rosen CF (eds): Osteoporosis, ed 3. Amsterdam, Elsevier, 2008, vol 2, pp 1012-1039.

29 Pietschmann P, Kudlacek S, Grishar J, Spitzauer S, Woloszczuk W, Willvonseder R, Peterlik M: Bone turnover markers and sex hormones in men with idiopathic osteoporosis. Eur J Clin Invest 2001;31:444-451.

30 Bouxsein ML: Bone quality: where do we go from here? Osteoporos Int 2003; 14:S118S127.
31 Seeman E: Bone quality: the material and structural basis of bone strength. J Bone Miner Res 2008;26:1-8.

32 Melton LJ 3rd, Riggs BL, van Lenthe GH, et al: Contribution of in vivo structural measurements and load/strength ratios to the determination of forearm fracture risk in postmenopausal women. J Bone Miner Res 2007; 22:1442-1448.

33 Seeman E: Periosteal bone formation: a neglected determinant of bone strength. N Engl J Med 2003;349:320-323.

-34 Beck TJ, Looker AC, Mourtada F, Daphtary MM, Ruff CB: Age trends in femur stresses from a simulated fall on the hip among men and women: evidence of homeostatic adaptation underlying the decline in hip BMD. J Bone Miner Res 2006;21:1425-1432.

35 Bonel HM, Lochmüller EM, Well H, Kuhn V, Hudelmaier M, Reiser M, Eckstein F: Multislice computed tomography of the distal radius metaphysis: relationship of cortical bone structure with gender, age, osteoporotic status, and mechanical competence. J Clin Densitom 2004;7:169-182.

36 Szulc P, Seeman E, Duboeuf F, Sornay-Rendu E, Delmas PD: Bone fragility: failure of periosteal apposition to compensate for increased endocortical resorption in postmenopausal women. J Bone Miner Res 2006; 21:1856-1863.

37 Gilsanz V, Boechat MI, Gilsanz R, Loro ML, Roe, TF, Goodman WG: Gender differences in vertebral sizes in adults: biomechanical implications. Radiology 1994;190:678-682.

38 Duan Y, Turner CH, Kim BT, Seeman E: Sexual dimorphism in vertebral fragility is more the result of gender differences in age-related bone gain than bone loss. J Bone Miner Res 2001; 16:2267-2275.

>39 Yang RS, Lin HJ, Chieng PU, Liu TK, Tsai KS: Estimated risk score for spine fracture in the specific bending activity of normal Taiwanese men and women. Spine 2005;30:22882292.

40 Bouxsein ML, Melton LJ 3rd, Riggs BL, Muller J, Atkinson EJ, Oberg AL, Robb RA, Camp JJ, Rouleau PA, McCollough CH, Khosla S: Age- and sex-specific differences in the factor of risk for vertebral fracture: a population-based study using QCT. J Bone Miner Res 2006;21:1475-1482.

41 Tinetti ME, Speechley M, Ginter SF: Risk factors for falls among elderly persons living in the community. N Engl J Med 1988;319: 1701-1707.

42 Greenspan SL, Myers ER, Maitland LA, Resnick NM, Hayes WC: Fall severity and bone mineral density as risk factors for hip fracture in ambulatory elderly. J Am Med Assoc 1994;271:128-133.

43 Smeesters C, Hayes WC, McMahon TA: Disturbance type gait speed affect fall direction and impact location. J Biomech 2001;34: 309-317. 
44 De Laet C, Kanis JA, Oden A, et al: Body mass index as a predictor of fracture risk: a meta-analysis. Osteoporos Int 2005; 16: 1330-1338.

-45 Hayes WC, Myers ER, Robinovitch SN, Van Den Kroonenberg A, Courtney AC, McMahon TA: Etiology and prevention of age-related hip fractures. Bone 1996;18:77-86.

-46 Bouxsein ML, Szulc P, Munoz F, Thrall E, Sornay-Rendu E, Delmas PD: Contribution of trochanteric soft tissues to fall force estimates, the factor of risk, and prediction of hip fracture risk. J Bone Miner Res 2007;22: 825-831.

47 Riggs BL, Parfitt AM: Drugs used to treat osteoporosis: the critical need for a uniform nomenclature based on their action on bone remodeling. J Bone Miner Res 2005;20:177184.

48 Blahos J: Treatment and prevention of osteoporosis. Wien Med Wochenschr 2007;157/ 23-24:589-592.
49 Orwoll E, Ettinger M, Weiss S, Miller P, Kendler D, Graham J, Adami S, Weber K, Lorenc R, Pietschmann P, Vandormael K, Lombardi A: Alendronate for the treatment of osteoporosis in men. N Engl J Med 2000; 343:604-610.

50 Reid DM, Adami S, Devogelaer JP, Chines AA: Risedronate increases bone density and reduces vertebral fracture risk within one year in men on corticosteroid therapy. Calcif Tissue Int 2001;69:242-247.

51 Ringe JD, Faber H, Farahmand P, Dorst A: Efficacy of risedronate in men with primary and secondary osteoporosis: results of a 1year study. Rheumatol Int 2006;26:427-431.

52 Seeman E: An exercise in geometry. J Bone Mineral Res 2002;17:373-380.

53 Wallace JM, Rajachar RM, Allen MR, Bloomfield SE, Robey PG, Young MF, Kohn DH: Exercise-induced changes in the cortical bone of growing mice are bone- and genderspecific. Bone 2007;40:1120-1127.

-54 Hulme PA, Krebs J, Ferguson SJ, Berlemann U: Vertebroplasty and kyphoplasty: a systematic review of 69 clinical studies. Spine 2006;31:1983-2001.
55 Parker MJ, Gillespie WJ, Gillespie LD: Hip protectors for preventing hip fractures in older people. Cochrane Database Syst Rev 2005;3:CD001255.

56 Oliver D, Conelly JB, Victor CR, Shaw FE, Whitehead A, Genc Y, Vanoli A, Martin FC, Gosney MA: Strategies to prevent falls and fractures in hospitals and care homes and effect of cognitive impairment: systematic review and meta-analyses. Br Med J 2007;334: 82-85.

57 Van Schoor NM, Smit JH, Bouter LM, Veenings B, Asma GB, Lips P: Maximum potential preventive effect of hip protectors. J Am Geriatr Soc 2007;55:507-510.

58 Holzer L, Holzer G: Die Bedeutung von Hüftprotektoren zur Prävention von hüftnahen Frakturen bei älteren Menschen. Wien Med Wochenschr 2007;157:381-387.

59 Laing AC, Robinovitch SN: Effect of soft shell hip protectors on pressure distribution to the hip during sideways falls. Osteoporos Int 2008;19:1067-1075. 